\title{
Evaluación del rendimiento y fenología de tres genotipos de tomate cherry (Solanum lycopersicum L.) bajo condiciones de invernadero
}

\section{Yield and phenology evaluation of three tomato cherry genotypes (Solanum lycopersicum L.) under greenhouse conditions}
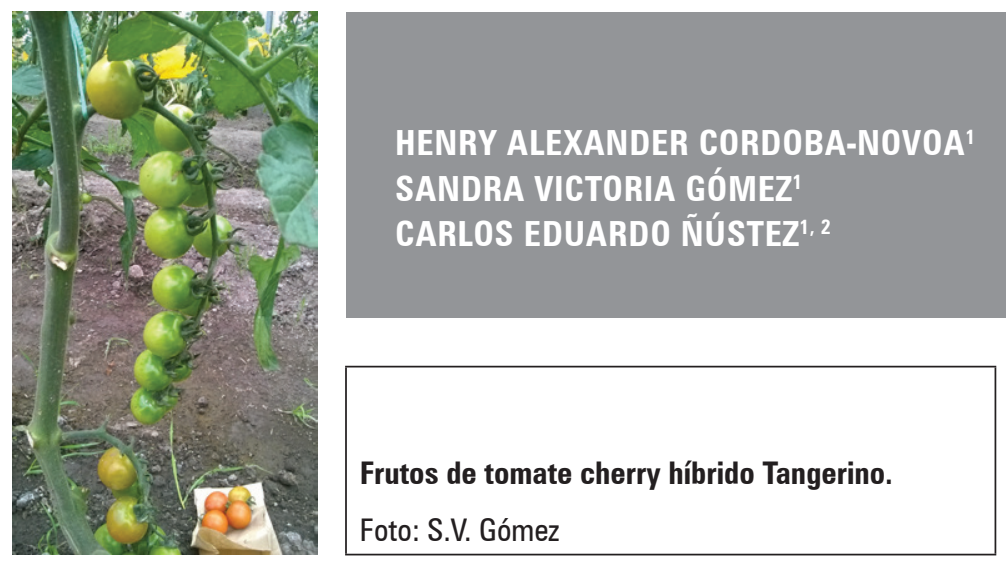

\section{RESUMEN}

La evaluación de características agronómicas de materiales genéticos disponibles en el mercado de tomate cherry es información relevante para la elección de materiales de siembra. Fueron evaluados tres genotipos de tomate cherry, dos híbridos: Tangerino y Tropical Cherry y la variedad Red Cherry. Las plantas fueron sembradas a una distancia de 0,5 por 1,0 m entre surcos y se evaluaron variables de fenología y las variables cuantitativas: número de flores por inflorescencia, frutos por planta, diámetro de frutos y rendimiento. En poscosecha se evaluó la colorimetría del fruto en dos temperaturas de almacenamiento durante 28 días, realizando mediciones cada 4 días. Los genotipos presentaron diferencias con respecto al número de flores por inflorescencia, número total de frutos por planta y rendimiento en $\mathrm{g} /$ planta, siendo Tangerino el que presentó la mejor respuesta en estas variables con un total de 25 flores por inflorescencia, 79 frutos por planta y $750 \mathrm{~g} /$ planta. En fenología no se presentaron diferencias en la duración de los estadios principales, fueron evidentes algunas variaciones de los estadios secundarios tales como aparición de brotes, órgano floral y maduración del fruto. El híbrido Tangerino fue el genotipo de mejor comportamiento por su rendimiento y maduración de frutos bajo las condiciones de evaluación.

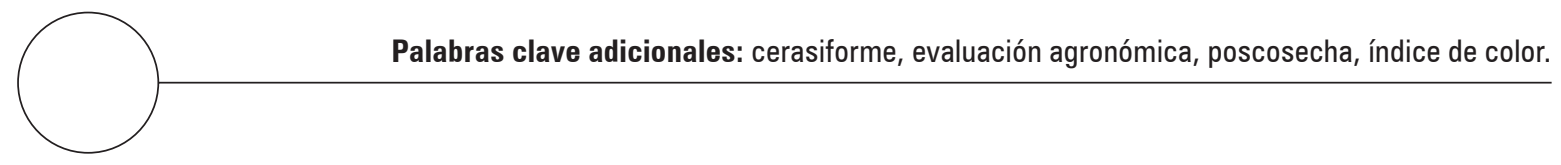

1 Facultad de Ciencias Agrarias, Universidad Nacional de Colombia, Bogotá (Colombia). ORCID Cordoba, H.A.: 0000-0002-2679-0456; ORCID Gómez, S.V.: 0000-0001-7950-0814; ORCID Ñústez, C.E.: 0000-0003-2000-022X

2 Autor para correspondencia.cenuztezl@unal.edu.co 


\section{ABSTRACT}

The evaluation of agronomic characteristics of genotypes available on the cherry tomato market is necessary for choosing the best material. Three cherry tomato genotypes (two hybrids: Tangerino and Tropical Cherry and the Red Cherry variety) were evaluated under greenhouse conditions. The plants were spaced at $0.5 \mathrm{~m}$ between plants and $1.0 \mathrm{~m}$ between rows. The phenology and quantitative variables, such as the number of flowers per inflorescence, fruits per plant, fruit diameter and yield, were determined. In the postharvest period, the fruit colourimetry was evaluated under two storage temperatures. The genotypes showed differences in the number of flowers per inflorescence, total number of fruits per plant and fruit yield, with Tangerino being the best in terms of these variables, with 25 flowers per inflorescence, 79 fruits per plant and $750 \mathrm{~g} / \mathrm{plant}$. In the phenology, there were no differences in the duration of the main stadiums, but there were small variations in the secondary stadiums, such as the appearance of branches, inflorescence and fruit ripening. The hybrid Tangerino had the best behavior in terms of yield and fruit ripening under the tested conditions.

Additional key words: cerasiforme, agronomic evaluation, postharvest, color index.

Fecha de recepción: 27-06-2017 Aprobado para publicación: 30-01-2018

INTRODUCCIÓN

El tomate es una de las hortalizas más consumidas en el mundo debido a los diversos tipos y formas que presenta para su consumo. Es una de las hortalizas de mayor valor económico (Marim et al., 2005) con 5,02 millones de hectáreas plantadas en el mundo y una producción total reportada de 170,7 millones de toneladas de frutos cosechados al año (FAOSTAT, 2014). En Colombia, la producción total de tomate para el año 2014 se estimó en 606.151 t y rendimiento de 33,98 tha-1 (FAO, 2014). Actualmente, la producción de tomate tipo cherry (Solanum lycopersicum var. cerasiforme) se ha incrementado notoriamente a nivel mundial, debido a su reporte de ser fuente importante de antioxidantes, de reducir el riesgo de contraer enfermedades crónicas y por su buen posicionamiento gastronómico para el consumo en ensaladas, salsas y cocteles, que lo hacen deseable en el mercado (Santiago et al., 1998; Giovannucci, 1999; CIT, 2000; Machado et al., 2003).

Una característica importante del tomate tipo cherry es su capacidad de aclimatarse adecuadamente en invernaderos fríos. Aunque presenta un mayor costo de mano de obra a cosecha, el buen precio que logra en el mercado lo compensa, por lo cual constituye una opción prometedora como hortaliza cultivable en condiciones de invernadero. Lo anterior, sumado a la aplicación de técnicas eficientes de agricultura limpia, otorga un valor agregado para la producción de este cultivo, notables ventajas a nivel comercial que se acentúan gracias a la creciente demanda del producto (García et al., 2004; Hernández, 2013).

Para responder a la demanda en aumento de tomate cherry, es necesario mejorar las condiciones de producción, entre las cuales debe considerarse la selección adecuada del material genético a utilizar. Un aspecto clave en la evaluación de genotipos es que cada uno presenta diferentes características en cuanto al crecimiento, tolerancia a problemas fitosanitarios, respuesta a las variables ambientales, rendimiento y cualidades del fruto (Abadie y Berretta, 2001). Un genotipo deseable para siembra deberá tener características sobresalientes, entre las que se incluyen: óptimo crecimiento y desarrollo, buen rendimiento denotado por número y peso de frutos, buena calidad del fruto, de buena adaptación al ambiente donde se pretende cultivar, aceptación en el mercado y buena poscosecha (Castellanos, 2009; Garzón, 2011). Sin embargo, hay que tener en cuenta que la respuesta esperada de un genotipo no es la misma bajo diferentes ambientes de cultivo, un comportamiento se tendrá en ambiente protegido y seguramente otro diferente en campo abierto, debido a que las variables climáticas son diferentes. También es posible que la respuesta de un genotipo sea diferente bajo ambientes protegidos distintos, debido a otras diferencias de cultivo tales como: la altitud, la localización geográfica, la 
tecnología de producción, las variaciones en la infraestructura del ambiente protegido, entre otros. Esta situación genera la recomendación de realizar ensayos de evaluación de los genotipos que se dispone para el sistema productivo, bien sea campo o invernadero, para escoger el de mejor comportamiento en dichas condiciones, sin olvidar las condiciones específicas del mercado de destino de la producción (Márquez et al., 2006; Castellanos, 2009).

Existe poca información relacionada con la evaluación de materiales genéticos de tomate tipo cherry, en particular orientada a la valoración de genotipos disponibles en el mercado y su producción bajo condiciones de invernadero. Es importante resaltar que el incremento del consumo y áreas de producción en los últimos años, principalmente con diversos materiales genéticos demanda evaluaciones agronómicas pertinentes. Generar información al respecto significa aportar en el conocimiento de este cultivo en el país y generar bases para posibles programas de mejoramiento. Por esta razón, el objetivo de este trabajo fue evaluar el rendimiento y fenología de tres genotipos de tomate cherry cultivados bajo invernadero en la Sabana de Bogotá e identificar el de mejores características agronómicas.

\section{MATERIALES Y MÉTODOS}

\section{Material vegetal y establecimiento del experimento}

El experimento se realizó en los invernaderos de la Universidad Nacional de Colombia, sede Bogotá, situada a 4³8'08" N y 7404'58' W a $2.640 \mathrm{msnm}$, con temperatura entre 14 y $30^{\circ} \mathrm{C}$ y humedad relativa que osciló entre 42 y $79 \%$. El experimento tuvo una duración de 6 meses de acuerdo con el ciclo del cultivo.

Los genotipos evaluados fueron los híbridos Tangerino y Tropical Cherry, junto con la variedad Red Cherry, todos de crecimiento indeterminado y adquiridos en el mercado de semillas de tomate cherry en Bogotá. Las semillas fueron embebidas durante $24 \mathrm{~h}$ en cajas Petri con papel absorbente húmedo, posteriormente se sembraron en semilleros con sustrato de turba Klasmann ${ }^{\circledR}$ de origen alemán. Transcurridos 38 $d$, las plantas fueron trasplantadas a una distancia entre surcos de $1 \mathrm{~m}$ y de $0,5 \mathrm{~m}$ entre plantas, para una densidad de siembra de 20.000 plantas/ha.

\section{Fenología y rendimiento}

Para cada genotipo se evaluó la fenología de acuerdo a los días después de trasplante (ddt) y según la escala BBCH para solanáceas (Feller et al., 1995). Se evaluaron características de momentos de aparición y desarrollo de órganos vegetativos (hojas verdaderas y brotes laterales) y reproductivos (botones, flores y frutos).

Se evaluaron las variables cuantitativas: número de flores por inflorescencia, diámetro promedio del fruto, número de frutos por planta, peso promedio de frutos cosechados y rendimiento por planta. La cosecha de los frutos se realizó a partir de los 88 ddt para los tres genotipos.

\section{Maduración poscosecha}

Se llevó a cabo la cosecha de frutos en los estados de maduración torneado (estado 3) y rojo claro (estado 5) de acuerdo con la escala de clasificación por color de tomate del Departamento de Agricultura de los Estados Unidos, USDA (UCDAVIS, 2016). La tabla 1 presenta la descripción de los estados de madurez según el cambio de coloración en la superficie del fruto.

Los frutos fueron almacenados bajo dos condiciones de temperatura; en cuarto frío $\left(5 \pm 1^{\circ} \mathrm{C}\right)$ y temperatura ambiente $\left(19^{\circ} \mathrm{C}\right.$ promedio); en ambos casos se determinó la colorimetría de los frutos cada cuatro días después de cosecha (ddc). El color de la epidermis de los frutos en cada estado de madurez se hizo con base en el sistema CIELab y con el uso del colorímetro digital Konica Minolta CR400. L indica la luminosidad, donde 0 es negro y 100 blanco; valores de (a) $<0$ indican tendencia hacia el verde y $>0$ hacia el rojo; (b) tiene el mismo rango pero valores $<0$ indican tendencia hacia el azul y $>0$ hacia el amarillo (Hernández et al., 2007). Se realizaron tres lecturas en la sección ecuatorial del fruto para determinar el índice de color (IC) con base en el índice propuesto por Hunter Lab IC $=\left(1000^{*} a\right) /\left(L^{*} b\right)$.

\section{Diseño experimental y análisis estadístico}

El experimento en campo se estableció bajo un diseño en bloques completamente al azar donde el tratamiento es genotipo, y para la evaluación de la maduración en poscosecha como un diseño completamente al azar con estructura factorial $3 \times 2$, donde el 
Tabla 1. Escala de clasificación por color de tomate del USDA (UCDAVIS, 2016).

\begin{tabular}{|c|c|l|}
\hline Estado & Nombre & \multicolumn{1}{|c|}{ Descripción } \\
\hline 1 & Verde & $\begin{array}{l}\text { La superficie del tomate es totalmente verde } \\
\text { en color. El tono de verde puede variar de } \\
\text { claro a oscuro. }\end{array}$ \\
\hline 2 & Quebrante & $\begin{array}{l}\text { Se presenta un quiebre en la coloración } \\
\text { verde del fruto por la aparición de pequeñas } \\
\text { coloraciones amarillas, rosas o verdes en no } \\
\text { más del 10\% de la superficie. }\end{array}$ \\
\hline 3 & Torneado & $\begin{array}{l}\text { Más del 10\% pero no más del 30\% de la } \\
\text { superficie presenta un cambio de coloración } \\
\text { de verde a amarillo, rosa, rojo o la combina- } \\
\text { ción de los mismos. }\end{array}$ \\
\hline 5 & Rojo claro & $\begin{array}{l}\text { Más del 30\% pero no más del 60\% de la } \\
\text { superficie del fruto muestra una coloración } \\
\text { rosa o rojiza. }\end{array}$ \\
\hline 6 & $\begin{array}{l}\text { Más del 60\% de la superficie del fruto mues- } \\
\text { tra una coloración rosa-rojiza o roja. No más } \\
\text { del de la superficie es de color rojo. }\end{array}$ \\
\hline Rojo & $\begin{array}{l}\text { Más del 90\% de la superficie del fruto mues- } \\
\text { tra una coloración roja. }\end{array}$ \\
\hline
\end{tabular}

primer factor correspondió al genotipo y el segundo factor a los ambientes de almacenamiento. El análisis estadístico se realizó con el programa SAS v. 9.4 (Statistical Analysis System) con un nivel de significancia de $P \leq 0,05$. Se validaron los supuestos de normalidad $\mathrm{y}$ de homocedasticidad en las variables por medio de las pruebas de Shapiro-Wilk y Bartlett, respectivamente. A los resultados obtenidos se les realizo análisis de varianza (ANOVA) y prueba de comparación múltiple de Tukey $(P \leq 0,05)$.

\section{RESULTADOS Y DISCUSIÓN}

\section{Desarrollo fenológico}

Se encontró que en los tres materiales genéticos evaluados el inicio de los estadios fenológicos principales ocurrió en los mismos ddt. Solo se encontraron variaciones en cuanto a los estadios fenológicos secundarios. Tangerino evidenció un desarrollo más precoz en el caso de formación de brotes laterales. Para los tres materiales evaluados, la aparición del órgano floral ocurrió en un periodo aproximado de $22 \mathrm{ddt}$, mientras que la floración se evidenció a los 44 ddt y la formación del fruto una vez transcurridos $74 \mathrm{ddt}$ (Figs.1, 2 y 3). No obstante, en cuanto a la aparición del órgano floral, floración y formación del fruto, 'Tropical Cherry' y 'Red Cherry' evidenciaron un comportamiento más precoz respecto a 'Tangerino, ya que alcanzaron los estadios secundarios dentro de estas etapas en un menor número de ddt (Figs. 1, 2 y 3). En cuanto a la maduración del racimo, 'Tropical Cherry' fue más precoz, completando la totalidad del proceso de maduración del racimo en $30 \mathrm{~d}$, respecto a los $41 \mathrm{~d}$ que tardaron los otros materiales evaluados.

En tomate común (Solanum lycopersicum) se reporta una duración promedio de la etapa vegetativa de entre 22 y $40 \mathrm{~d}$. Luego del trasplante, el tomate continúa en esta etapa por 30 a $35 \mathrm{~d}$ más y a los 50 o $60 \mathrm{~d}$ inicia la floración, aunque se reporta que estos periodos dependen ampliamente de la variedad (Pérez et al., 2002). Por otra parte, se ha reportado que para el caso del tomate tipo cherry, el tiempo transcurrido desde el trasplante a campo hasta la aparición de la primera inflorescencia, corresponde a un periodo de 3 a 4 semanas (Fullelove et al., 1998). Lo anterior concuerda con el tiempo aproximado hasta la aparición del órgano floral que se encontró en el experimento para los tres materiales genéticos estudiados. En el caso de la floración, los tres materiales evaluados presentaron un inicio tardío de la misma respecto a lo reportado para el tomate común, ya que ésta se produjo a los 44 ddt, es decir aproximadamente a los $82 \mathrm{~d}$ después de siembra.

En plantas de tomate la floración comienzan hacia los $45 \mathrm{~d}$ después de la emergencia en el campo y sus flores perfectas son agrupadas en inflorescencias tipo racemoso. Se afirma que en la floración del tomate, tanto cultivado como silvestre, el genotipo es uno de los factores relevantes para la respuesta de esta variable (Nuez, 1995; Restrepo et al., 2008). En evaluación realizada a la colección de Solanum sección Lycopersicum de la Universidad Nacional de Colombia Sede Palmira, se encontró importante variación en la variable de inicio de floración, dos introducciones de S. lycopersicum var. cerasiforme fueron las más precoces con $41 \mathrm{~d}$ después de la emergencia para el inicio de la floración (Restrepo et al., 2008). Los tiempos de floración encontrados por Restrepo et al. (2008) coinciden con los hallados en el experimento, lo que permite concluir que si bien se encontró un retraso en la época de floración para estos materiales respecto al tomate común, las épocas de aparición del órgano floral y floración sí coinciden con lo reportado para el tomate tipo cherry en otros estudios realizados. 


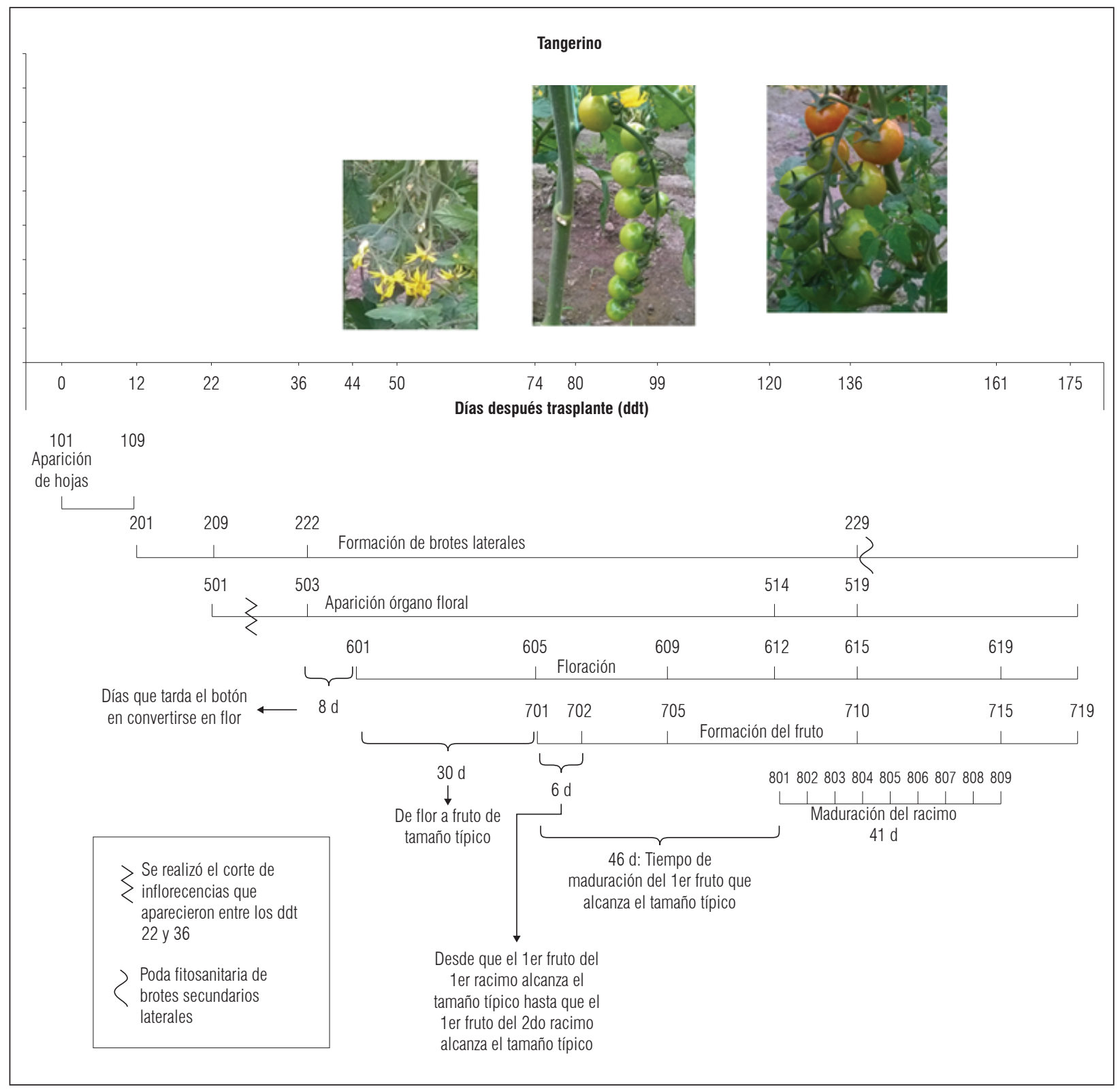

Figura 1. Desarrollo fenológico del híbrido Tangerino.

La etapa reproductiva, se extiende por unos 32 a 40 $\mathrm{d}$ antes de la cosecha, la cual se inicia entre los 62 y $75 \mathrm{ddt}$. Esta etapa tarda entre 30 y $40 \mathrm{~d}$ y se caracteriza porque se detiene el crecimiento de la planta e inicia el crecimiento y maduración de los frutos (CATIE, 1990; Pérez et al., 2002). En el presente estudio la etapa reproductiva inició en el día 74 después de trasplante para el caso de los tres materiales, lo cual concuerda con el intervalo de tiempo aproximado establecido para el inicio de este estadio fenológico en plantas de tomate.

\section{Flores por inflorescencia}

Se encontraron diferencias significativas entre 'Tangerino' y 'Red Cherry', mientras que 'Tropical Cherry' no difirió de los otros genotipos en el punto máximo de aparición de flores (Fig. 4). El rango máximo observado para flores por inflorescencia osciló entre 5 y 16 en los genotipos evaluados, siendo 'Tangerino' el que alcanzó mayor número de flores por inflorescencia (16). De forma general, los tres genotipos evaluados mostraron un decrecimiento progresivo en los 


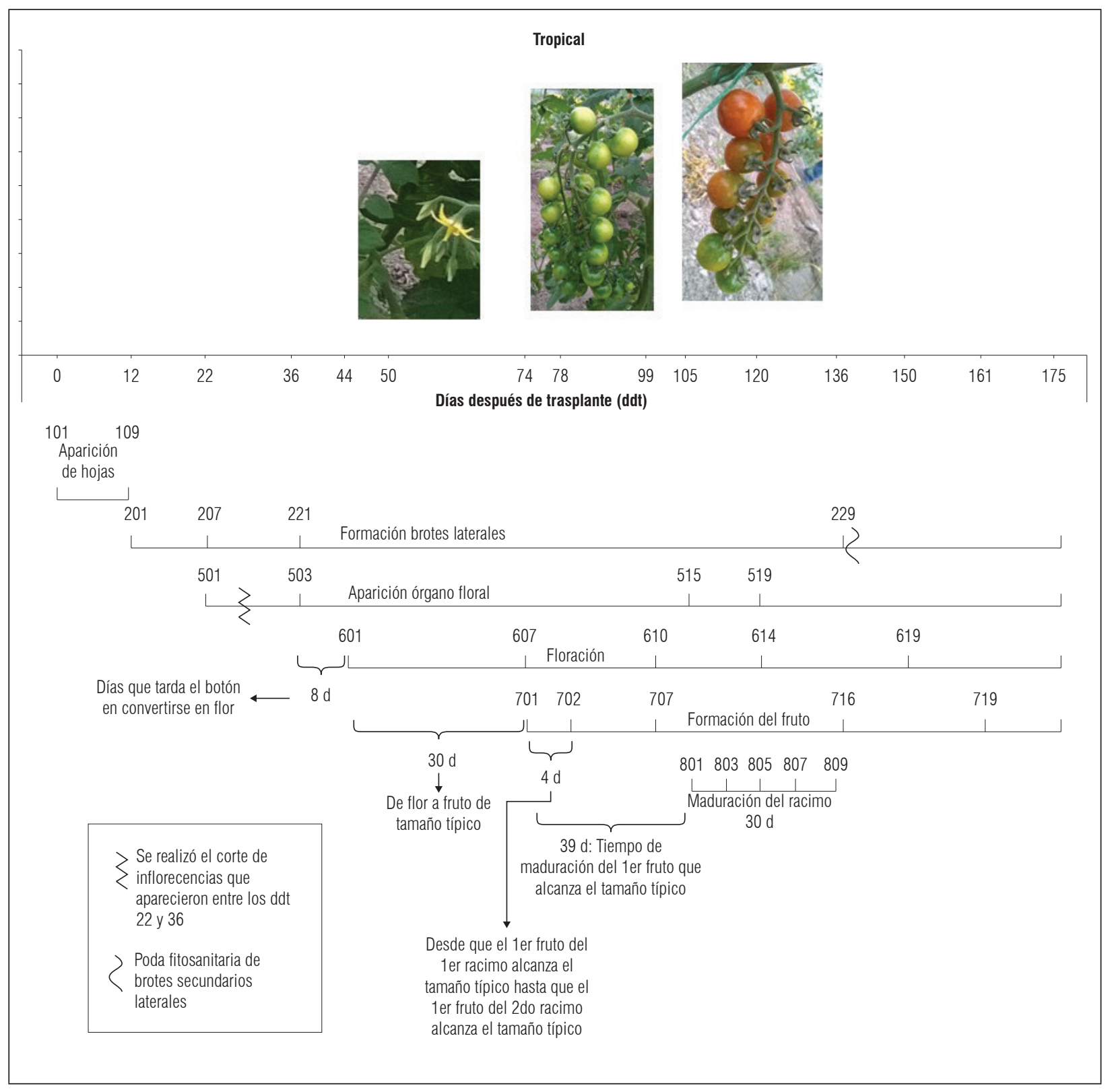

Figura 2. Desarrollo fenológico del híbrido Tropical Cherry.

valores de esta variable a través del tiempo. Autores como Lobo y Medina (1994) evaluaron la variabilidad morfológica del tomate tipo cherry, encontrando que el número de flores por inflorescencia varía entre 4 y 20, lo cual se aproxima notablemente al intervalo encontrado para los tres genotipos evaluados. Ceballos y Vallejo (2012) encontraron que en un $80 \%$ de las variedades, se produjo una relación directa entre un mayor número de flores por inflorescencia y un número más alto de frutos.
Otros autores como Boada et al. (2010) encontraron diferencias significativas en cuanto al número de flores por inflorescencia entre 26 introducciones de tomate cherry evaluadas. Estos autores encontraron una amplia variabilidad en los valores obtenidos, los cuales oscilaron entre 17,4 y 177 flores por inflorescencia. Estos valores superan ampliamente los hallados en el presente trabajo y evidencian la magnitud en la cual puede variar el número de flores por inflorescencia entre genotipos de tomate cherry. De otro 


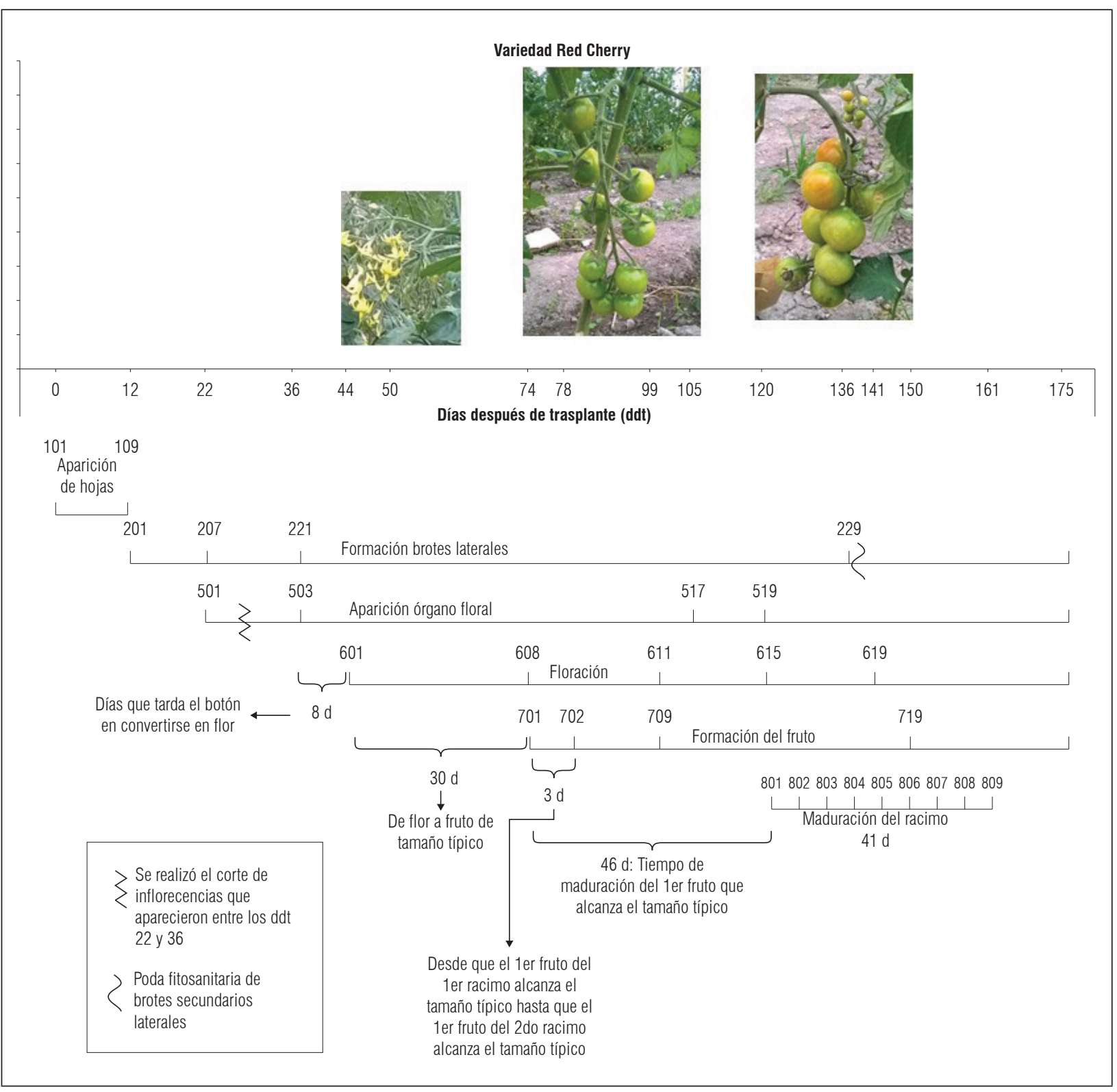

Figura 3. Desarrollo fenológico de la variedad Red Cherry.

lado, Pratta (2003) encontró un rango de 6 a 15 flores por racimo, que concuerda casi de manera exacta con los datos obtenidos en el presente estudio. Boada et al. (2010) argumentan que el número de tallos o ejes presentes en la planta influye en la cantidad de racimos totales, lo que consecuentemente incide en el número total de flores por racimo, encontrándose una mayor producción de éstas a mayor número de tallos en la planta, aunque con un tamaño de fruto y duración del cultivo menores.

\section{Diámetro y número promedio de frutos}

Los frutos de los tres genotipos evaluados no presentaron diferencias significativas con respecto al diámetro promedio. Los valores oscilaron entre 11,1 y 23,0 $\mathrm{mm}$, siendo 'Tangerino' el de mayor diámetro promedio (Tab. 2). Los resultados obtenidos para Tangerino son similares a los reportados por Pulvento et al. (2008) quienes evaluaron el efecto del estrés hídrico en diferentes cultivares de tomate cherry, obteniendo 


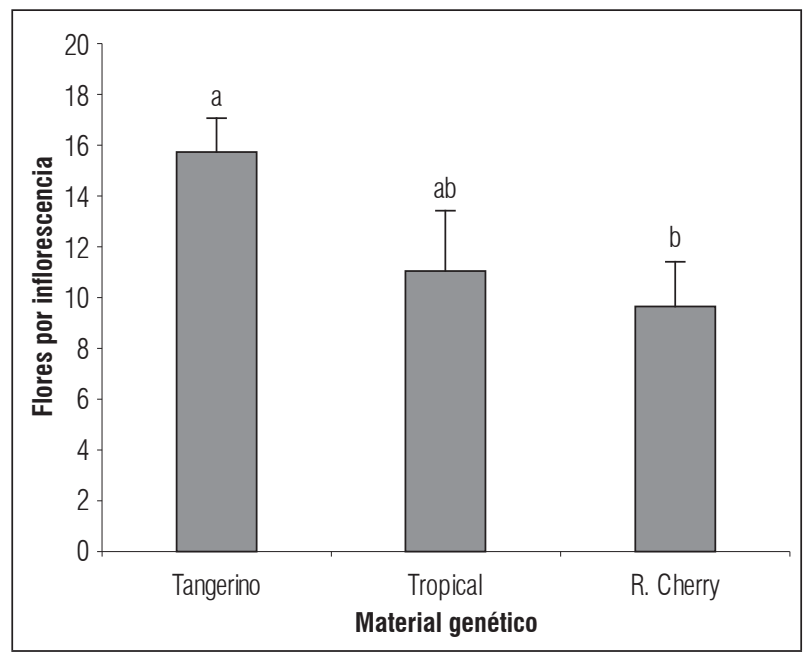

Figura 4. Flores por inflorescencia en tres genotipos de tomate tipo cherry en el punto máximo de aparición de flores (99 ddt). Promedios con letras distintas indican diferencia significativa según la prueba de Tukey $(P \leq 0,05)$. Las barras de error indican el error estándar.

frutos con diámetros cercanos y por encima de los 20 $\mathrm{mm}$ para el tratamiento control. Por otra parte, Cachoeira et al. (2005) en la comparación morfológica de tres sistemas de cultivo de tomate cherry encontraron diámetros promedio de los frutos cercanos a 24 mm y Agudelo et al. (2011) reportan diámetros entre 20 y $30 \mathrm{~mm}$. De acuerdo con lo anterior, el diámetro encontrado para 'Tangerino' se sitúa en este intervalo, pero los encontrados para 'Tropical Cherry' y 'Red Cherry' son menores. No obstante, Nuez (1999) describe que los tomate cherry se caracterizan por producir frutos de tamaño muy pequeño y variable (10 a $30 \mathrm{~mm}$ de diámetro), lo cual permite decir que los resultados encontrados para el tamaño promedio del fruto es propio de cada genotipo bajo las condiciones de evaluación.

En el número total de frutos por planta, 'Tangerino' fue el que presentó el valor más alto, seguido por 'Tropical Cherry' y 'Red Cherry' (Tab. 2). Este resultado concuerda con lo reportado por Techawongstien et al. (2009), Ceballos y Vallejo (2012) y Hossain et al. (2010; 2015) quienes encuentran amplios rangos de variación en el número de frutos por planta como característica dependiente del material genético entre los que se contemplan los valores encontrados para los tres genotipos evaluados en el presente estudio.
Tabla 2. Diámetro promedio del fruto (DPF) y número de frutos de los tres genotipos evaluados.

\begin{tabular}{|l|c|c|}
\hline \multicolumn{1}{|c|}{ Genotipo } & DPF $(\mathrm{mm})$ & $\begin{array}{c}\text { Número de frutos } \\
\text { por planta }\end{array}$ \\
\hline Tangerino & $20,3 \pm 2,74 \mathrm{a}$ & $79 \pm 5 \mathrm{a}$ \\
\hline Tropical & $15,0 \pm 3,53 \mathrm{a}$ & $52 \pm 5 \mathrm{~b}$ \\
\hline Variedad & $14,5 \pm 3,38 \mathrm{a}$ & $51 \pm 8 \mathrm{~b}$ \\
\hline
\end{tabular}

Promedios con letras distintas indican diferencia significativa según la prueba de Tukey $(P \leq 0,05)$.

\section{Rendimiento de fruto}

Con respecto al rendimiento de fruto en $\mathrm{g} /$ planta se presentaron diferencias significativas entre los genotipos; 'Tangerino' fue el que presentó el mayor rendimiento promedio $(750,5 \mathrm{~g} /$ planta), seguido por 'Tropical Cherry' (508,6 g/planta) y finalmente 'Red Cherry' (199,2 g/planta) (Fig. 5). El mayor rendimiento reportado para 'Tangerino' puede estar relacionado con un número superior de flores por inflorescencia respecto a los otros dos materiales, como se encontró en el presente estudio. En cuanto a 'Tropical Cherry' y 'Red Cherry', Casierra y Aguilar (2008) reportan que existe una variación en la acumulación de sólidos solubles totales en los frutos, según el estado de desarrollo en el cual se cosechan, el cual a su vez depende de la variedad de tomate evaluada, existen por tanto híbridos para los cuales el punto de corte no afecta esta característica, mientras que para otros sí es determinante. Por tal razón, la variación en el rendimiento de 'Tropical Cherry' y 'Red Cherry', aun cuando el número de frutos y el diámetro de los mismos fue similar, pudo deberse a diferencias en la acumulación de sólidos solubles en los estados de desarrollo del fruto, debidas a diferencias en el comportamiento de cada material.

Resultados similares a los obtenidos para 'Tangerino' fueron reportados por Hossain et al. (2010, 2015) quienes obtuvieron un rendimiento por planta de 776 g. Monge-Pérez (2015) destaca que el rendimiento es una característica que presenta mucha variabilidad y que es altamente dependiente del material genético, las condiciones ambientales, la presencia de plagas y enfermedades y las prácticas de manejo como densidad de siembra y podas realizadas. Por otra parte, Truffault et al. (2016) encontraron rendimientos máximos para plantas de tomate cherry bajo invernadero cercanos a los $400 \mathrm{~g} /$ planta. De acuerdo con esto, es evidente la alta variabilidad que se puede presentar 


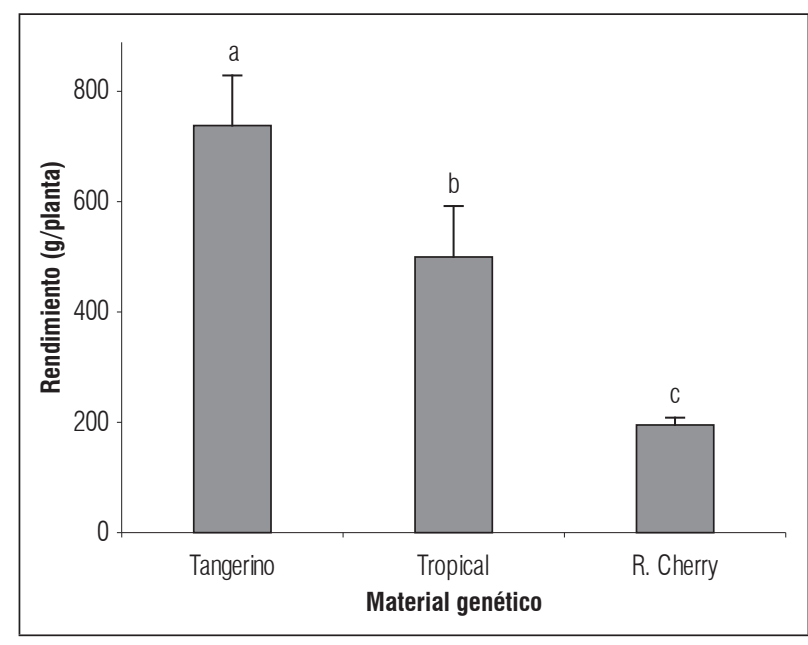

Figura 5. Rendimiento de tres genotipos de tomate cherry. Promedios con letras distintas indican diferencia significativa según la prueba de Tukey $(P \leq 0,05)$. Las barras de error indican el error estándar.

en el rendimiento de diferentes genotipos evaluados, hecho que concuerda con los resultados obtenidos por Techawongstien et al. (2009) quienes en la evaluación de 17 materiales genéticos de tomate cherry obtuvieron rendimientos que oscilaron entre los 14,2 hasta los $1.049 \mathrm{~g} /$ planta.

En el ciclo de cultivo se encontraron dos picos de producción; a los 149 y 176 ddt para los tres genotipos evaluados, siendo Tangerino el que presentó valores superiores para la variable en el tiempo, e igualmente, tuvo los picos de mayor proporción en estos dos periodos mencionados, alcanzando hasta $150 \mathrm{~g} /$ planta de producción a los 149 ddt (Fig. 6). 'Red Cherry' mostró los menores valores de esta variable durante todo el experimento, alcanzando tan solo un máximo aproximado de $40 \mathrm{~g} /$ planta a los 149 ddt. Tropical se situó en un nivel intermedio de producción, alcanzando un máximo de $80 \mathrm{~g} /$ planta para los frutos cosechados a los 149 ddt (Fig. 6).

\section{Maduración en poscosecha}

El índice de color (IC) establece la variación de la coloración del fruto con base en los valores de a, b y L (Hernández et al., 2007). El estado de madurez de los frutos al momento de la cosecha e inicio de almacenamiento afectó el índice de color, al presentarse los valores más bajos para los frutos cosechados en verde, independientemente del material y ambiente de almacenamiento (Fig. 7). El incremento en el IC a lo

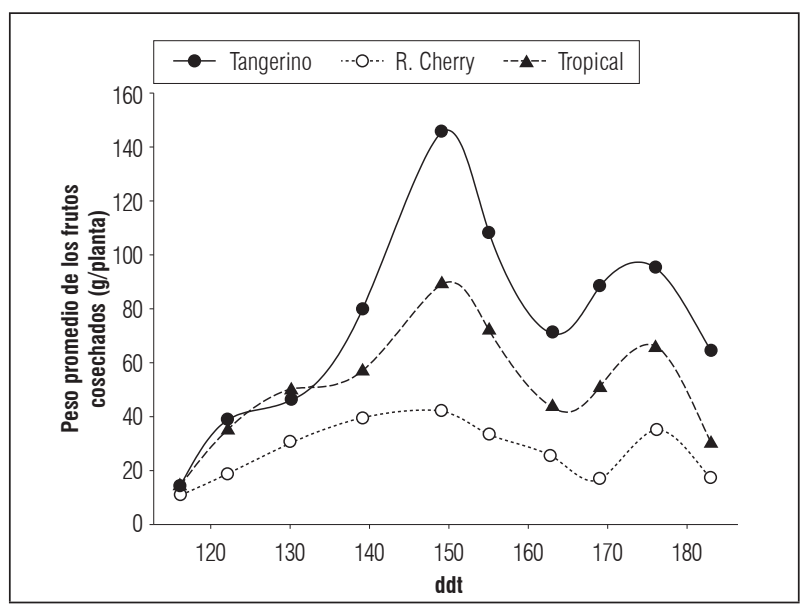

Figura 6. Peso promedio de los frutos cosechados por planta a lo largo del ciclo de cultivo para cada uno de los genotipos evaluados.

largo del tiempo tanto para los frutos cosechados en rojo claro como los cosechados en torneado, indican que a pesar del momento de cosecha y condiciones de almacenamiento, los frutos de tomate llevan a cabo un proceso de maduración en poscosecha. Este proceso de maduración es el resultado de una serie de cambios físicos y químicos que tienen lugar gracias a la producción de etileno que se da por la naturaleza climatérica del fruto (Galietta et al., 2005). Con respecto al cambio de color, Padrón et al. (2012) argumentan que a medida que los pigmentos rojos inician su síntesis como parte del proceso de maduración en el fruto, los valores de L decrecen y por tanto el IC aumenta.

La temperatura de almacenamiento tuvo efecto significativo sobre el IC de los dos tipos de frutos cosechados (Figs. 7A y B). Almacenamiento a $5 \pm 1^{\circ} \mathrm{C}$ causó una disminución promedio de 5,4 unidades para los frutos rojo claro y de 4,5 para los frutos torneados. En el caso de los frutos rojo claro se obtuvo un valor máximo de IC de 15,8 a temperatura ambiente y de 9,6 a $5 \pm 1^{\circ} \mathrm{C}$. Para los frutos torneados, el máximo IC alcanzado a temperatura ambiente fue 14,2 y a $5 \pm 1^{\circ} \mathrm{C}$ fue 4,7 . Estos resultados muestran que la menor temperatura permitió reducir la síntesis de pigmentos y parte del proceso de maduración. Al respecto, se ha reportado que una maduración a temperatura inferior a $12^{\circ} \mathrm{C}$, considerada sub-óptima para la síntesis de licopeno, permite la acumulación de $\beta$-caroteno en los cromoplastos (Shewfelt et al., 1988; López-Camelo et al., 2003). Esta acumulación diferencial de pigmentos, sumada a una reducción en la degradación de clorofila y la acumulación de carotenoides por el efecto de 
las bajas temperaturas sobre la actividad de enzimas como la clorofilasa (Tietel et al., 2012) resultan en la obtención de frutos anaranjados o amarillentos.
Entre los materiales genéticos evaluados se evidenciaron diferencias significativas en el IC independiente de la temperatura de almacenamiento (Figs. 7C y D).

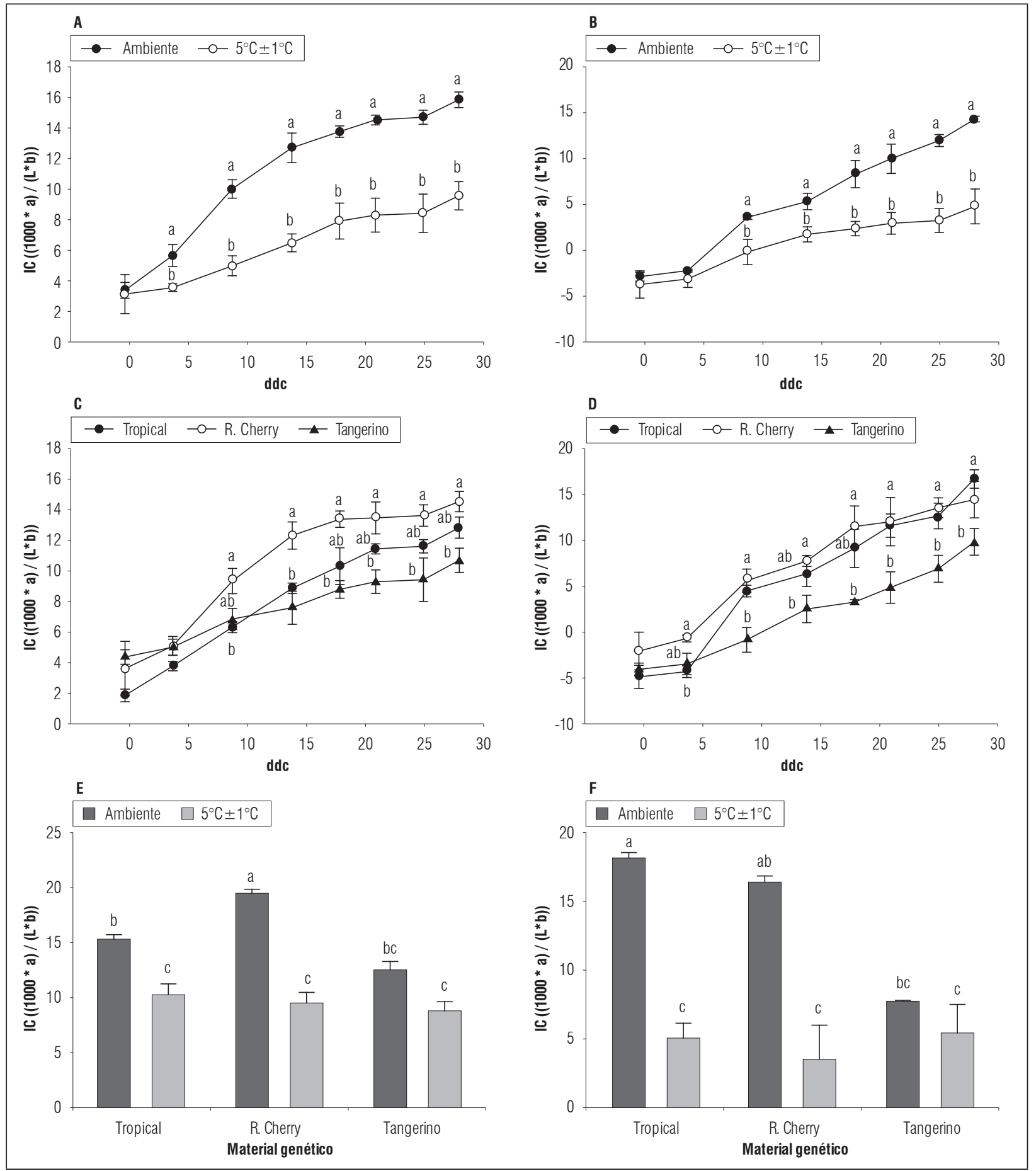

Figura 7. Efecto de la temperatura de almacenamiento $(A$ y $B)$, del material genético $(C$ y $D)$ y la combinación de factores ( $E$ y F) sobre el IC de frutos cosechados en estado rojo claro $(A, C$ y $E)$ y frutos cosechados en estado torneado $(B, D$ y $F)$. Promedios con letras distintas indican diferencia significativa según la prueba de Tukey $(P \leq 0,05)$. Las barras de error indican el error estándar. 
A partir de los 9 ddc y hasta los $28 \mathrm{ddc}$ (periodo de evaluación), la variedad Red Cherry presentó los IC más altos y significativamente mayores a los presentados por el híbrido Tangerino. Para el caso del híbrido Tropical Cherry se obtuvieron valores medios para el IC respecto a los otros dos materiales genéticos, sin diferencias significativas con los mismos. Estos resultados sugieren que 'Red Cherry' presenta un proceso de maduración más rápido en comparación con 'Tangerino' donde el proceso de maduración, denotado por el cambio en la coloración, es más lento.

El efecto combinado de la temperatura y el material genético sobre los frutos cosechados, tanto en verde como en rojo, mostró que los mayores IC se presentaron en los frutos almacenados a temperatura ambiente y que estos fueron significativamente mayores al de los frutos almacenados a $5 \pm 1^{\circ} \mathrm{C}$, este es el caso de 'Tropical Cherry' y 'Red Cherry' (Figs. 7E y F). Dicho comportamiento fue consistente a partir de los $9 \mathrm{ddc}$ hasta el final del periodo de evaluación (28 ddc). Para 'Tangerino' no hubo una reducción significativa del IC cuando los frutos se almacenaron en refrigeración independientemente del estado de madurez de cosecha. Los resultados de la interacción de los factores concuerdan con el evidenciado para el efecto individual de la temperatura y el material genético. Para el caso de 'Tangerino', la ausencia de diferencias entre frutos rojo claro y torneados almacenados a temperatura ambiente y a $5 \pm 1^{\circ} \mathrm{C}$, indican que la refrigeración no juega un papel crucial en proceso de maduración de los frutos de este material, si se considera únicamente el IC como indicador del proceso de maduración.

Las alteraciones en los cambios fisiológicos que tienen lugar en el fruto cuando este es almacenado a baja temperatura permite explicar la disminución del IC de los frutos de Tropical y Red Cherry. El comportamiento evidenciado para Tangerino, es decir, un menor IC en las diferentes condiciones de almacenamiento puede deberse a características propias del híbrido. Durante la maduración del tomate, inicialmente se sintetiza fitoeno (incoloro), para dar lugar a $\beta$-caroteno (anaranjado, amarillo pálido), licopeno (rojo), xantofilas y carotenoides hidroxilados (amarillos). De acuerdo con esto, el híbrido tangerino puede presentar una menor síntesis natural de pigmentos, en especial pigmentos rojos en el fruto, o una mayor síntesis de los pigmentos de tonalidades amarillas (Shewfelt et al., 1988; López-Camelo et al., 2003).

La ausencia de diferencias entre los ambientes de almacenamiento para los frutos de 'Tangerino' sugiere que este híbrido cuenta con características genéticas específicas que permiten que el proceso de maduración sea mucho más lento y por tanto que la vida en poscosecha sea mayor. Sin embargo, es necesario investigaciones posteriores que permitan elucidar los mecanismos que este genotipo presenta y que interfieren directamente con la maduración del fruto.

\section{CONCLUSIONES}

Los materiales genéticos evaluados evidenciaron un comportamiento similar en cuanto a fenología, presentando únicamente diferencias en el comienzo de los estadios secundarios 207 a 209, 514 a 517 y 705 a 719. Fue evidente un comportamiento más precoz en el híbrido Tangerino respecto a la formación de brotes laterales. 'Tangerino' fue el material genético que presentó mayor producción de flores por inflorescencia, mayor peso promedio de frutos cosechados, mayor número de frutos por planta y consecuentemente una respuesta de rendimiento superior respecto a los otros dos materiales genéticos. En poscosecha, a temperatura ambiente los frutos del genotipo Red Cherry presentaron un cambio más veloz a tonalidad rojiza seguido por 'Tropical Cherry' y finalmente por 'Tangerino'. En temperatura de $5 \pm 1^{\circ} \mathrm{C}$, los dos primeros materiales fueron similares en maduración. 'Tangerino' presentó una maduración de fruto similar y más lenta en ambas condiciones de almacenamiento. Estos resultados permiten afirmar que el genotipo más indicado para producción bajo las condiciones evaluadas es el híbrido Tangerino.

Conflicto de intereses: el manuscrito fue preparado y revisado con la participación de los autores, quienes declaran no tener algún conflicto de interés que coloquen en riesgo la validez de los resultados aquí presentados.

\section{REFERENCIAS BIBLIOGRÁFICAS}

Abadie, T. y A. Berretta. 2001. Caracterización y evaluación de recursos fitogenéticos. En: Estrategia en recursos fitogenéticos para los países del Cono Sur. PROCISUR, e IICA. Montevideo, Uruguay, http://www.procisur. org.uy/data/documentos/21.pdf., consulta: junio de 2016.

Agudelo, A., N. Ceballos y F. Orozco. 2011. Caracterización morfológica del tomate tipo cereza (Solanum lycopersicum L.). Agron. 19(2), 44-53.

Boada, M., J. Mejía, N. Ceballos y F. Orozco. 2010. Evaluación agronómica de treinta introducciones de tomate 
silvestre tipo cereza (Solanum lycopersicum L.) Agron. 18(2), 59-67.

Cachoeira, S., A. do Espiritu Santo, C. Bona y R. Sossela de Freitas. 2005. Comparative morphological analysis of cherry tomato fruits from three cropping systems. Sci. Agric. 62(3), 296-298. Doi: 10.1590/ S0103-90162005000300015

Casierra, F. y O. Aguilar. 2008. Calidad de frutos de tomate (Solanum lycopersicum L.) cosechados en diferentes estados de madurez. Agron. Colomb. 26(2), 300-307.

Castellanos, J. (ed). 2009. Manual de producción de tomate en invernadero. Intagri SC, Celaya, Guanajuato, México.

CATIE. 1990. Guía para el manejo integrado de plagas del cultivo de tomate. Proyecto Regional Manejo integrado de plagas. Centro Agronómico Tropical de Investigación y Enseñanza. Turrialba, Costa Rica.

Ceballos, N. y F. Vallejo. 2012. Evaluating the fruit production and quality of cherry tomato (Solanum lycopersicum var. cerasiforme). Rev. Fac. Nal. Agr. Medellín 65, 6593-6604.

CIT. 2000. Sumario Información Tecnológica. Información Tecnológica 11(5), 192.

FAO. 2014. Anuario estadístico de la FAO. En: FAO (Organización de las Naciones Unidas para la Alimentación y la Agricultura), http://www.fao.org/3/a-i3592s; consulta: febrero de 2015.

FAOSTAT. 2014. FAO Statistical pocketbook 2014. En: http://www.fao.org/documents/card/en/c/ 383d384a-28e6-47b3-a1a2-2496a9e017b2; consulta: diciembre de 2016.

Feller, C., H. Bleiholder, L. Buhr, H. Hack, M. Hess, R. Klose, U. Meier, R. Stauss, T. Van Den Boom y E. Weber. 1995. Phänologische Entwicklungsstadien von Gemüsepflanzen: II. Fruchtgemüse und Hülsenfrüchte. Nachrichtenbl. Deut. Pflanzenschutzd. 47, 217-232.

García R., A. Berenguer, M. Tormo, M. Sanchez, J. Quiros, C. Navarro, R. Arnaud, M. Dorronsoro, M. Chirlaque, A. Barricarte, E. Ardanaz, P. Amiano, C. Martinez, A. Agudo y C. Gonzalez. 2004. Dietary sources of vitamin C, vitamin E and specific carotenoides in Spain. Br. J. Nutr. 91(6), 1005-1011. Doi: 10.1079/BJN20041130

Galietta, G., F. Harte, D. Molinari, R. Capdevielle y W. Diano. 2005. Aumento de la vida útil poscosecha de tomate usando una película de proteína de suero de leche. Rev. Iberoamer. Tecnol. Postcosecha 6(2), 117-123.

Garzón, J. 2011. Caracterización y evaluación morfoagronómica de la colección de tomate tipo cherry de la Universidad Nacional de Colombia sede Palmira. Tesis de maestría. Facultad de Ciencias Agropecuarias, Universidad Nacional de Colombia, Palmira, Colombia.

Giovannucci E. 1999. Tomatoes, tomato-based products, lycopene, and cancer: review of the epidemiologic literature. J. Natl. Cancer Inst. 91(4), 317-331. Doi: 10.1093/jnci/91.4.317
Hernández, M., O. Martínez y J. Fernández-Trujillo. 2007 Behavior of arazá (Eugenia stipitata Mc Vaugh) fruit quality traits during growth, development and ripening. Sci. Hortic. 111(3), 220-227. Doi: 10.1016/j. scienta.2006.10.029

Hernández, S. 2013. Estudio del comportamiento de distintos tipos de sustratos de lana de roca, en respuesta al aumento de oxígeno disuelto en la solución nutritiva respecto a la producción y calidad de un cultivo de tomate tipo "cherry pera". En: Universidad de Almería, http://repositorio.ual.es:8080/jspui/handle/10835/2641\#.V2Af8bt97IU; consulta: abril de 2016.

Hossain, M., V. Strezov, K. Chan y P. Nelson. 2010. Agronomic properties of wastewater sludge biochar and bioavailability of metals in production of cherry tomato (Lycopersicon esculentum). Chemosphere 78(9), 1167. 1171. Doi: 10.1016/j.chemosphere.2010.01.009

Hossain, M., V. Strezov y P. Nelson. 2015. Comparative assessment of the effect of wastewater sludge biochar on growth, yield and metal bioaccumulation of cherry tomato. Pedosphere 25(5), 680-685. Doi: 10.1016/ S1002-0160(15)30048-5

Lobo, M. y C. Medina. 1994. Phenotypic evaluation of Latin American tomato cultivars. p. 26. En: Tomato genetics cooperative report. Cornell University, Nueva York, USA.

López-Camelo, A., P. Gómez y F. Artés. 2003. Use of a* and $b^{*}$ colour parameters to assess the effect of some growth regulators on carotenoid biosynthesis during postharvest tomato ripening. Acta Hort. 599, 305-308. Doi: 10.17660/ActaHortic.2003.599.36

Machado, J., L. Braz y G. Grilli. 2003. Desempenho de produção de cultivares de tomateiro tipo Cereja em diferentes espaçamentos (CD). Hortic. Bras. 21(2), 356-356.

Marim, B., D. Silva, M. Guimarães y G. Belfort. 2005. Sistemas de tutoramento e condução do tomateiro visando produção de frutos para consumo in natura. Hortic. Bras. 23(4), 951-955. Doi: 10.1590/ S0102-05362005000400018

Márquez, C., P. Cano, M. Chew, A. Moreno y N. Rodríguez. 2006. Sustratos en la producción orgánica de tomate cherry bajo invernadero. Rev. Chapingo Ser. Hortic. 12(2), 183-188. Doi: 10.5154/r.rchsh.2005.01.001

Monge-Pérez, J. 2015. Evaluación de 60 genotipos de tomate (Lycopersicon esculentum Mill.) cultivados bajo invernadero en Costa Rica. InterSedes 16(33), 2215-2458.

Nuez, F. 1995. El cultivo del tomate. Mundi-Prensa. Madrid, España.

Nuez, F. 1999. El cultivo del tomate. $2^{\text {da }}$ ed. Ediciones Mundi-Prensa, Madrid, España.

Padrón, C., G. Padrón, A. Montes y R. Oropeza. 2012. Determinación del color en epicarpio de tomates (Lycopersicum esculentum mill.) con sistema de visión 
computarizada durante la maduración. Agron. Costar. 36(1), 97-111.

Pérez, J., G. Hurtado, V. Aparicio, O. Argueta y M. Larín. 2002. Guía técnica cultivo de tomate. Centro Nacional de Tecnología Agropecuaria y Forestal, San Salvador.

Pratta, G., L. Cánepa, R. Zorzoli y L. Picardi. 2003. Efecto del germoplasma silvestre sobre caracteres de interés agronómico en híbridos intra e interespecíficos del género Lycopersicon. Rev. Inv. Fac. Cienc. Agrar. 3(3), 13-21

Pulvento, C., M. Riccardi, R. d'Andria, A. Lavini y D. Calandrelli. 2008. Effects of deficit irrigation on two cherry tomato cultivars in hilly areas. Options Méditerranéennes 84, 177-184.

Fullelove, G., R. Wright, N. Meurant, J. Barnes y R. O’Brien. 1998. Tomato information kit. National Library of Australia, Queensland, Australia.

Restrepo E., F. Vallejo y M. Lobo. 2008. Fenología de la floración en tomate cultivado y especies silvestres relacionadas. Acta Agron. 57(2), 89-94.

Santiago J., M. Mendoza y F. Borrego. 1998. Evaluación de tomate (Lycopersicum esculentum Mill.) en invernadero: criterios fenológicos y fisiológicos. Agron. Mesoamer. 9(1), 59-65.
Shewfelt, R., C. Thai y J. Davis. 1988. Prediction of changes in color of tomatoes during ripening at different constant temperatures. J. Food Sci. 53(5), 1433-1437. Doi: 10.1111/j.1365-2621.1988.tb09293.x

Techawongstien, S., C. Lapjit, K. Khamkula, J. Komnoo, W. Yokla, T. Prasomsuay y P. Suemanotham. 2009. Varietal improvement of cherry tomato for good quality and high yield. Khon Kaen Agr. J. 37, 51-60.

Tietel, Z., E. Lewinsohn, E. Falliky R. Porat. 2012. Importance of storage temperatures in maintaining flavor and quality of mandarins. Postharvest Biol. Tecnol. 64(1), 175 182. Doi: 10.1016/j.postharvbio.2011.07.009

Truffault, V., N. Gest, C. Garchery, A. Florian, A. Fernie, H. Gautier y R. Stevens. 2016. Reduction of MDHAR activity in cherry tomato suppresses growth and yield and MDHAR activity is correlated with sugar levels under high light. Plant Cell Environ. 39, 1279-1292. Doi: $10.1111 /$ pce.12663

UCDAVIS (University of California, Davis). 2016. Maturity \& quality. En: UCDAVIS, Postharvest Technology: Maintaining Produce Quality \& Safety, http://postharvest.ucdavis.edu/pfvegetable/TomatoPhotos/?repository $=30014 \& \mathrm{a}=83755$; consulta: junio de 2016 . 